\title{
Review of: "Multiple phage resistance systems inhibit infection via SIR2-dependent NAD+ depletion"
}

\author{
Jingmin Gu
}

Potential competing interests: The author(s) declared that no potential competing interests exist.

This is a very interesting research. Authors found that DSR2, a defense-associated sirtuins (DSR) in Bacillus subtilis, becomes an active NADase when stimulated by phage tail tube proteins, directly leading to deplete the cell of NAD+ and abort phage propagation. In addition, this research also found there are phage derived anti-DSR2 proteins which can bind and repress DSR2. This is another example of the arms race between bacteriophages and bacteria. 